\title{
The Cheapest and Easiest Way to Make Platelet-rich Plasma Preparation
}

\author{
Han Moi Choi ${ }^{1}$, Soon Heum Kim ${ }^{1}$, \\ Cheol Keun Kim ${ }^{1}$, Hyun Gon Choi ${ }^{2}$, \\ Dong Hyeok Shin ${ }^{2}$, Ki Il Uhm², \\ Dongin $\mathrm{Jo}^{1}$
}

${ }^{1}$ Department of Plastic and Reconstructive Surgery, Konkuk University School of Medicine, Chungju; ${ }^{2}$ Department of Plastic and Reconstructive Surgery, Konkuk University School of Medicine, Seoul, Korea

The authors did not receive any funding or benefits from any companies related directly or indirectly to the subject of this paper.

This article was presented at the 70th congress of the Korean society of plastic and reconstructive surgeons on November 9-11, 2012.

No potential conflict of interest relevant to this article was reported.
Background Platelet-rich plasma (PRP) has been advocated as a way to introduce increased concentrations of growth factors and other bioactive molecules to injured tissues in an attempt to optimize the local healing environment. Many methods for PRP preparation have been introduced. Despite variations in the volume of whole blood taken and the efficacy of the platelet concentration, the main objective of PRP preparation is to obtain sufficient platelet concentration in the finally processed autologous plasma. We have been making our own internal primitive PRP preparation, which is safe and aseptic, using simple tubes and a centrifugal separator at the outpatient department base.

Methods Twenty cc of whole blood was collected and $10 \mathrm{cc}$ of blood was added to each of two bottles, followed by addition of $1.5 \mathrm{cc}$ adenosine-citrate-dextrose-acid solution to each bottle. Then, centrifugal separation was performed at 4,000 RPM for $15 \mathrm{~min}$ utes. Then, the buffy coat layer was aspirated using a $10 \mathrm{cc}$ syringe equipped with a spinal needle. Platelet activation was initiated by addition of $\mathrm{CaCl}_{2}$ and botropase.

Results We were successful in attaining PRP, which was three folds and six folds concentrated compared with the initial platelet count of whole blood.

Conclusions Our protocol is economical and only requires a few simple procedures for preparation of PRP. We expect the protocol to be applied to clinical trials without significant cost of time and money.

Keywords Platelet-rich plasma, Platelet count, Economics

\section{INTRODUCTION}

Platelet-rich plasma (PRP) is the abbreviation for PRP, which is made by centrifugation of whole blood. PRP consists of highly concentrated platelets and a small volume of plasma. It has been widely used since the 1970s, and is known for its effect on the wound healing process. The healing process was accelerated from the cellular level. Alpha granules of platelets contain various growth fac-

Received: Dec 17, 2014 Revised: Feb 12, 2015 Accepted: Feb 13, 2015 Correspondence: Dongin Jo Department of Plastic and Reconstructive Surgery, Konkuk University School of Medicine, 82 Gugwon-daero, Chungju 380-704, Korea. E-mail: drdijo@naver.com

Copyright (C) 2015 The Korean Society for Aesthetic Plastic Surgery.

This is an Open Access article distributed under the terms of the Creative Commons Attribution Non-Commercial License (http://creativecommons.org/licenses/by-nc/3.0/) which permits unrestricted non-commercial use, distribution, and reproduction in any medium, provided the original work is properly cited. www.e-aaps.org tors, such as platelet-derived growth factor (PDGF), transforming growth factor beta (TGF- $\beta$ ), vascular endothelial growth factor (VEGF), insulin-like growth factor-1 (IGF-1), hepatocyte growth factor (HGF), and fibroblast growth factor (FGF), which accelerate mitosis, vasculogenesis, and differentiation $[1,2]$. They are released when platelets are activated [3].

PRP is widely used in the field of plastic surgery, acting as a catalyst for the wound healing process, as well as increasing the success rate of lipotransfer, and restoration of skin tone. PRP, which also has an antimicrobial effect, could be applied on infected wounds, decreasing the need for antibiotics [4].

Although PRP treatment is well known for its useful effects, the high cost made it less accessible to both doctors and patients (Table 1).

We designed a cost-effective and reliable method for preparation of concentrated PRP. The results for use of our method were assessed by platelet concentration, time, cost, and possibility of contamination. 
Table 1. Price, time, cost, and concentration of several Platelet-rich plasma products. The information was obtained from the manufacturer's product literature

\begin{tabular}{|c|c|c|c|c|c|}
\hline Method & Manufacture & $\begin{array}{c}\text { Disposable } 1 \text { kit price } \\
\text { (USD) }\end{array}$ & $\begin{array}{l}\text { Hardware list price } \\
\text { (USD) }\end{array}$ & $\begin{array}{l}\text { Time of preparation } \\
\text { (min) }\end{array}$ & $\begin{array}{c}\text { Approximate } \\
\text { concentration factor }\end{array}$ \\
\hline KUH method & Konkuk University Hospital & 9.53 & 352.21 & $\begin{array}{l}15 \text { (1st) } \\
30 \text { (2nd) }\end{array}$ & $\begin{array}{l}3 \text { (1st) } \\
6 \text { (2nd) }\end{array}$ \\
\hline Cell saver based system & www.fresenius-kabi.com & $75-175$ & 10,000 & 20 & $4-5$ \\
\hline Biomet system GPS II & www.biomet.com & 700 & 16,000 & 30 & 3.2 \\
\hline Autologel system & www.cytomedix.com & 325 & 5,000 & $1-2$ & $2-4$ \\
\hline Genesis CS & www.vet-stem.com & 1,550 & 11,500 & 16 & $10 \pm 3$ \\
\hline Genesis CS-2 & www.vet-stem.com & 135 & 2,000 & 15 & 5.4 \\
\hline $\begin{array}{r}\text { Harvest smart } \\
\text { Prep2 BMAC }\end{array}$ & www.harvesttech.com & 420 & 9,950 & 16 & 4 \\
\hline
\end{tabular}

\section{METHODS}

This study was approved by the institutional review board of our hospital. Written informed consent was obtained from each patient. Between January and December of 2013, 30 patients were treated with one cycle of PRP preparation and 28 patients were treated with two cycles of PRP preparation.

Blood samples were harvested from 58 volunteers (20 male and 38 female), who provided informed consent before conduct of the investigation. All patients had external wounds (decubitus ulcer, diabetic foot foot and few acute wounds). From each patient, $21 \mathrm{cc}$ of blood was harvested. In each sample, $10 \mathrm{cc}$ of blood was transferred to a glass bottle containing $1.5 \mathrm{cc}$ adenosine-citrate-dextroseacid (ACD-A) solution (AJU Pharm Co., Ltd., Seoul, Korea); 1 cc of blood was used for complete blood cell count. The value of the platelet count was measured using XE-2100 (Sysmex Corp. Kobe, Japan). In the case of the two cycle preparation, we harvested $41 \mathrm{cc}$ of whole blood and performed the same cycle twice (Fig. 1).

We searched for the appropriate numerical value of rpm and centrifugal time. Based on our experience, the minimum rpm for separation of the buffy coat layer was 4,000 RPM, and the best result was shown at 4,000 RPM and 15 minutes. At those times and RPM, platelet-poor plasma (PPP), PRP, and buffy coat layers were the most well visualized.

In our centrifuge (PLC-02, Gemmy Industrial Corp., Taipei, Taiwan), the maximum rpm value is 4,500 and the maximum value of G-force is 2422 (Fig. 2). By our formula, radius of our centrifuge is calculated as $106.79 \mathrm{~mm}$. We substitute the value of R and RPM, 106.79 and 4,000. Calculated G-force is 1,913.68 $\mathrm{g}$ (The formula for calculating the value of $\mathrm{G}$ force is as follows: $\mathrm{G}$-force $=1.12 \times \mathrm{R}$ $\times(\mathrm{RPM} / 1,000)^{2}$. $\mathrm{R}$ is the radius of rotation length measured in millimeters). By substituting the value of R and RPM, the G-force of the centrifuge could be calculated using this equation, and the researcher would be able to attain the necessary value of RPM of each centrifuge.

However, it is recommended that the researcher perform several tests in order to find the best value for rpm and the time for centrifugation in other centrifuges. We believe that the optimal rpm and time for centrifuge may differ slightly depending on each machine.

Bottles were centrifuged for 15 minutes at 4,000 RPM. Then, the buffy coat layer and some of the serum layer were harvested together using a $10 \mathrm{cc}$ syringe equipped with a spinal needle. We then prepared PRP needed for activation of the release of a-granule contents and for maintenance of secreted proteins on the wound site. Activation solution was made with $3 \%$ calcium and botropase in the proportion of three to one. The amount of activation solution was a tenth part of PRP. The activated PRP was drawn into a $1 \mathrm{cc}$ syringe, and the PRP was injected around the wound at the subcutaneous level.

A whole blood test was initiated and compared to the platelet count. The immediate platelet count after harvest was established as a baseline. We obtained the concentration rate by comparing the platelet count obtained by the first and second segregation with the baseline count.

Patients with thrombocytopenia (platelet count under 200,000 per microliter) or coagulation disorder were excluded. The wound size, patients' age, and underlying condition were diverse and excluded for those reasons.

All patients were tested for human immunodeficiency virus (HIV), hepatitis, venereal disease research laboratory (VDRL), and several other diseases prior to the process in order to guarantee the safety of the surgeon and the people involved with the procedure. All procedures were performed under the sterile condition. 

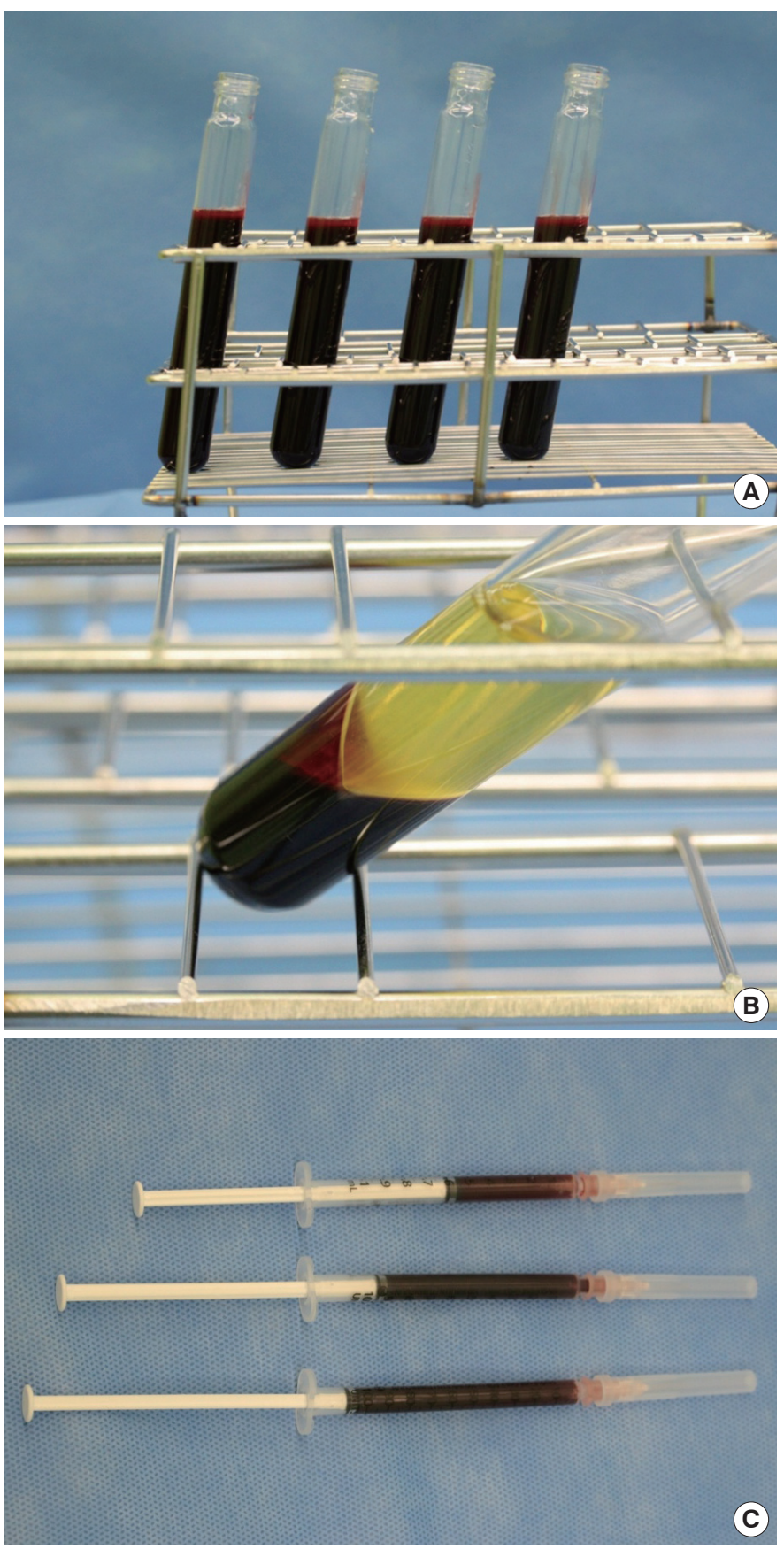

Fig. 1. (A) was harvested whole blood mixed with adenosine-citratedextrose-acid (ACD-A) solution. (B) shows whitish colored buffy coat layer. (C) is Platelet-rich plasma (PRP) preparation.

The lab data were analyzed using SPSS software, version 18.00 (SPSS Inc., Chicago, IL, USA). The concentration ratio from each centrifugation group was subjected to normality testing. Normally distributed data could be assessed by independent two sample test. The normality test was used to determine whether our test data have normal distribution. The value of $\mathrm{P}<0.05$ was regarded as a statistically significant value.

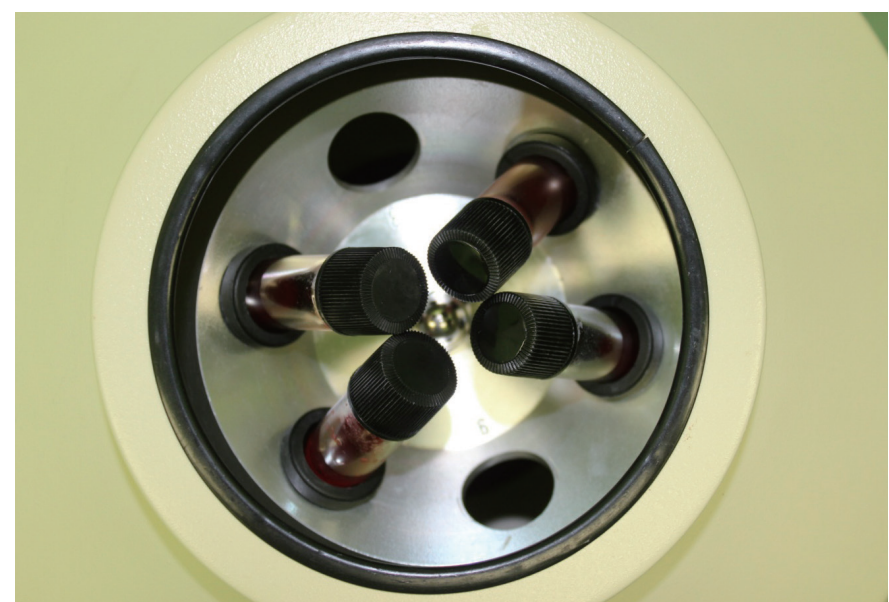

Fig. 2. Internal view of PLC-02 centrifuge.

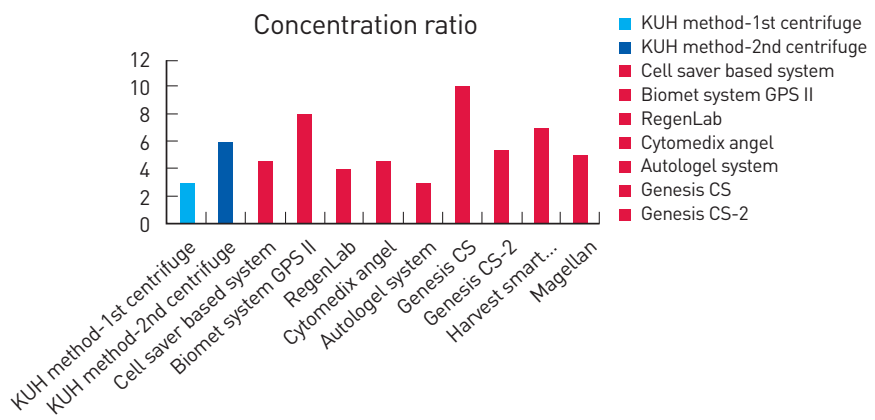

Fig. 3. Platelet concentration ratio between whole blood and Platelet-rich plasma (PRP) preparation. The x-axis represents the method of PRP preparation. The $y$-axis represents the concentration of platelets between whole blood and PRP preparation.

We obtained the value of the other PRP's platelet concentration and the cost of product from the information given on the website and the catalog from the companies.

\section{RESULTS}

Among the products, our method showed competent and stable results for platelet concentration compared with whole blood (Fig. 3). After the first cycle, approximately 2-3 folds of platelet concentration was checked. After the second cycle, approximately six folds of platelet concentration was measured. Statistical analysis was performed for determination of platelet concentration between PRP preparations and whole blood (Fig. 4).

The P-values assessed by Kolmogorov-Smirnov and ShapiroWilk test were higher than 0.05 , indicating that the data show normal distribution. Then, the data were assessed by independent two samples test. The significance probability was 0.00 for both groups. Therefore, the data from the one cycle group and the two cycle group were statistically different. 


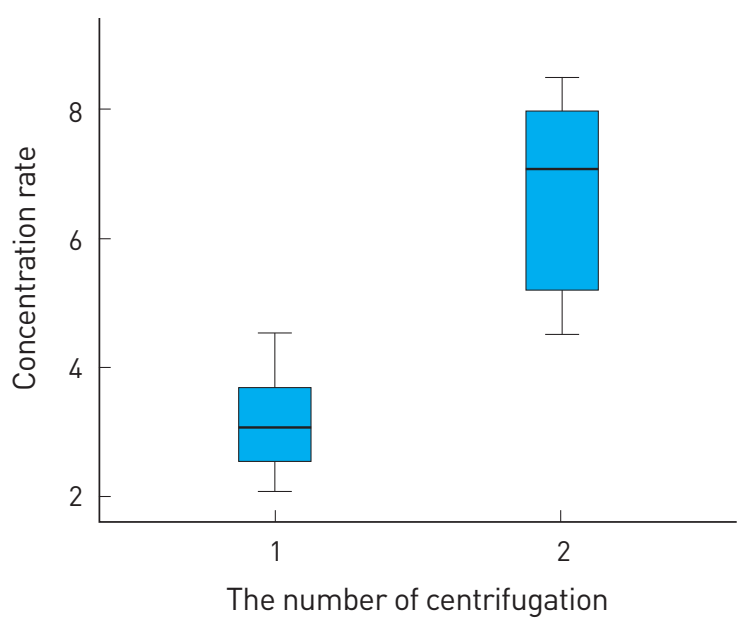

Fig. 4. Analysis of platelet concentration rate between baseline and Platelet-rich plasma (PRP) preparation. Analysis was performed using SPSS 18.0.

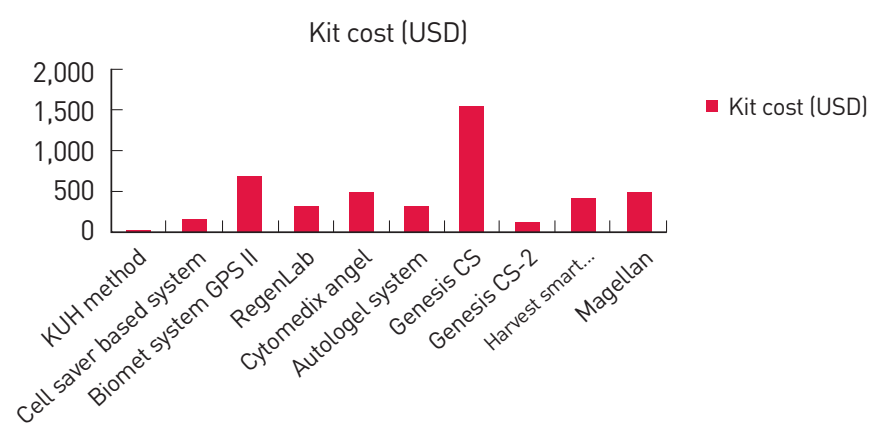

Fig. 5. Total cost of the disposable kit. The x-axis represents the method of Platelet-rich plasma (PRP) preparation. The $y$-axis represents the total cost (USD).

The time spent in the entire procedure was analyzed excluding the time taken for drape and harvesting. The time taken for centrifugation was 15 minutes and separation took between 1 to 2 minutes. The entire procedure took 17 minutes in the one centrifugation group and 35 minutes in the two centrifugation group.

Total price of one disposable kit was within 10 USD. The setting price of the centrifuge machine was within 400 USD. The total price was less than that for any existing methods (Fig. 5, Table 2).

The entire procedure was performed in the operation room of the outpatient department. Microbial culture was performed for each procedure, however, microbial growth was not observed.

\section{Case 1}

A 40-year-old man sustained a 3rd degree burn to the right ear, with multiple necrotic change on helix and tragal area (Fig. 6A). Preoperative photo was taken 2 weeks after the injury. There are necrotic tissue on preauricular area, tragus and upper helical rim. Methicillin-resistant Staphylococcus aureus (MRSA) was isolated
Table 2. Cost and materials of our method [13-15]

\begin{tabular}{lccc}
\hline Materials & Number & Purpose & Cost (USD) \\
\hline 1 cc syringe & 1 & Injection & 0.05 \\
10 cc syringe & 2 & Separation & 0.15 \\
50 cc syringe & 1 & Harvesting & 0.21 \\
Spinal needle & 1 & Separation & 2.94 \\
Botropase & 1 & Activation & 1.76 \\
$3 \%$ CaCl$_{2}$ & 1 & Activation & 0.27 \\
ACD-A solution & 2 & Anticoagulant & 3.92 \\
Glass bottle & 6 & Collection & 0.23 \\
Centrifuge PLC-02 (Gemmy & 1 & Separation & 352.21 \\
Industrial Corp., Taipei, Taiwan) & & & \\
Total & & 361.74 &
\end{tabular}

ACD-A, adenosine-citrate-dextrose-acid.

and antibacterial agent was administrated by IV (Fig. 6B). The picture was taken 3 weeks from the injury. Necrotic tissue became well demarcated. Debridement and split thickness skin graft was performed (Fig. 6C). The picture was taken 1 week after the operation. Tragus and lower portion of antihelix showed positive healing state, but necrotic tissue was still remaining at the upper portion of the helical rim and the antihelix. PRP was injected around the necrotic area and the unstable area at this time (Fig. 6D). The picture was taken 1 week after the PRP injection. The raw surface of the superior antihelix and the helical rim became smaller in size (Fig. $6 \mathrm{E}, \mathrm{F})$. The picture was taken 2 weeks after the PRP injection. The ear was well healed state. Well healed state of posterior aspect of the helical rim.

\section{DISCUSSION}

Platelets are non-nucleate, round-shaped blood cells that serve a variety of important functions, with involvement in hemostasis, inflammation, angiogenesis, wound healing process, and antimicrobial host defense. The role of platelets in the wound healing process is initiation and modification of the healing process, which involves multiple proteins and cytokines.

PRP is used in various fields, not only in aesthetic areas, but also in the wound healing process. It can be used to accelerate the healing process of muscle, tendon, and cartilage. For chronic wounds, PRP can improve the healing process and reduce the time until the wounds were covered by secondary intention. In aesthetic areas, PRP can improve skin turgor, as well as reduce wrinkling and skin pigmentation. In an animal study, the ability of PRP to increase the survival of the autologous fat graft was demonstrated. In an acute wound, PRP could accelerate secondary intention. Local administration of a concentrated state of growth factors and cytokines has been shown to augment the healing effect of local connective tissue. Many in vitro studies have shown a dose-response relationship 

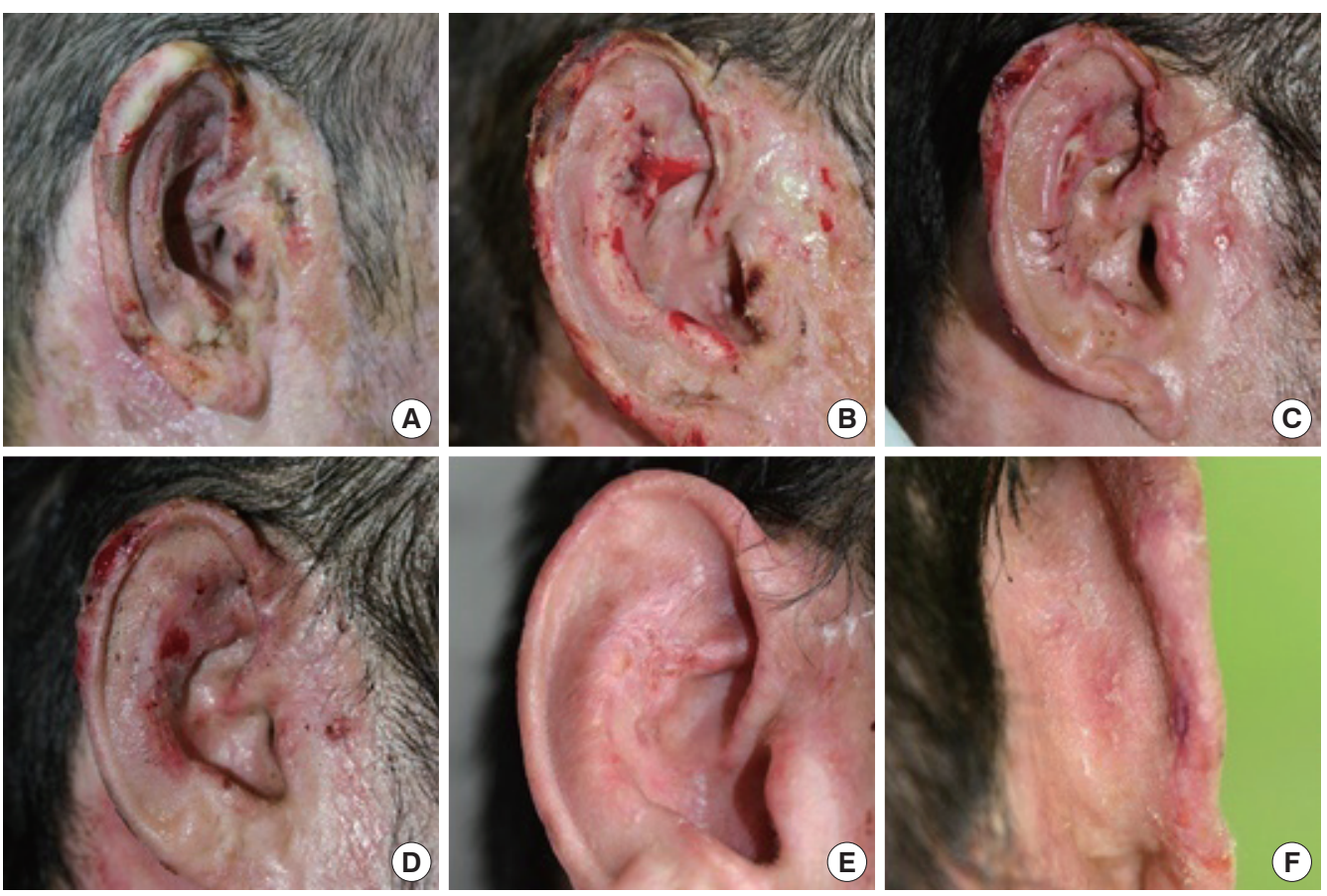

Fig. 6. Case 1. 3rd degree burn on right ear. with the healing effect according to the growth factors contained in PRP. However, those relationships are not linear. Concentration of growth factors is important, however, receptors of target cells are also important. When receptors of the cell surface were fully occupied, no additional effect was observed.

Eppley et al. [5] designed a study on the relationship between platelet concentration and growth factor level. When platelets were concentrated eight times more than normal level, the levels of PDGF$\mathrm{BB}, \mathrm{TGF}-\beta 1, \mathrm{VEGF}$ and endothelial growth factor were elevated as well. However, the level of elevation was different for each individual.

In our investigation, platelet concentration ratio by one cycle separation showed an average of three folds and two cycle separation showed an average of six folds from the baseline. Our preparation solution contains a high concentration of platelets, thus, we estimated that our PRP preparation contains sufficiently concentrated growth factor. Therefore, we can expect that our PRP preparation has a sufficient therapeutic effect for treatment.

There are three main advantages of using our method. The first advantage of our procedure is the reliable supply of platelet concentration. Data from the first centrifugation and second centrifugation were analyzed using the SPSS 18.00 program. Our data included fewer than 50 cases; therefore, non-parametric tests were performed to check the distribution of data. Both groups showed normal distribution, indicating that our method has shown a stable outcome.

Since our data showed normal distribution, it was possible to compare the mean value and the concentration ratio between groups from the parametric tests. We used the t-test for comparison between the one cycle group and the two cycle group. Values of sig- nificance for both groups were defined as higher than 0.05 . T-test and independent samples tests were performed. For the independent samples test, value of significance for Levene's test was 0.000, meaning that both groups had different variance. As a result, means of both groups showed a statistically significant difference.

The second advantage of using our method is that it is time efficient. One separation of PRP took 16 minutes. Two cycle separation took 20 minutes more than the one cycle separation, so that choice of one cycle or two cycles depends on the preference of the researcher.

Finally, using our method is economical and cost effective. From an economical point of view, the cost of one disposable kit is less expensive compared with other methods. The cost of setting was more cost-efficient compared with the existing methods. Any facilities who wish to test our method could use a pre-equipped centrifuge. The only requirement is to calculate the $\mathrm{G}$ force of the centrifugal machine. In Korea, PRP is not covered by the National Health Insurance. Currently, the Korean government's official stand on PRP treatment is that while they recognize the stability of the treatment, when it comes to the effectiveness of the medical treatment, more outcomes should be gathered for investigation. Thus, any cost with the exception of aesthetic purposes, the fee for treatment will not be covered by insurance. Under these circumstances, our PRP preparation method will support the use of PRP for clinical treatment.

Many investigators have designed a simple method for making a PRP preparation. Merques et al. [6] devised a simple method similar to ours. By the way, our method could use PRP preparation in step 1 . When a more concentrated PRP preparation is needed, pro- 
ceed to step 2.

When leukocytes were removed from the PRP intentionally, it is known as pure-PRP (P-PRP) or P-PRP, PRP containing leukocytes is defined as leukocyte-PRP (L-PRP) or L-PRP. Our PRP preparation contains some white blood cell (WBC), thus, L-PRP was used for research. Bielecki et al. [7] suggested that L-PRP inhibits the growth of Staphylococcus aureus and Escherichia coli in vitro. Another study reported by Moojen et al. [8] found that L-PRP has a greater antimicrobial activity than PRP alone. Even nonunion and delayed union fractures complicated by infection were treated successfully with L-PRP [9]. Improvement of functional recovery and analgesic requirement was reported $[10,11]$. Li et al. [12] reported that L-PRP had a statistically significant antimicrobial effect on MRSA infected rabbit.

The entire procedure consists of two phases. The first phase is the centrifugation of whole blood and separation of the PRP layer. The second phase is activation of PRP and injection to the target area. The amount of separated PRP obtained was approximately 6 cc for one-cycle preparation and approximately 1.5 cc for two-cycle preparation from $40 \mathrm{cc}$ whole blood used. Activation of PRP was generally achieved by calcium and bovine thrombin. Activated PRP initiates clot formation and exocytosis of alpha granules. In particular, bovine thrombin could make antibody of clotting factor $\mathrm{V}, \mathrm{XI}$ and could risk coagulopathy state. Therefore, to avoid use of bovine thrombin, PRP was activated by $3 \%$ calcium chloride and botropase solution. Activation was done just before the injection. Following activation, injection was administered rapidly before PRP clotting. Remaining PRP was used in a gel state for maintenance of a contact state between the wound bed and PRP solution.

There are concerns regarding infection during the procedures. These concerns are that blood clot is a good media for microbacterium, so that infection could occur easily. In contrast to $\mathrm{pH}$ value of blood clot from normal blood, which is 7.0 to 7.2 , the $\mathrm{pH}$ value of PRP is 6.5 to 6.7. Therefore, clots made from PRP have potency of bacterial growth restriction. In this investigation, we performed the entire process at the outpatient department base. Bacterial isolation was performed during each process, however, bacterial growth was not found.

The platelet concentration rate became stable and increased in proportion to the number of times centrifuge was performed. By our method, mean concentration rate was approximately three folds for the one cycle group and six folds for the two cycle group. This is an excellent concentration rate vis-à-vis commercial products. Even in terms of time taken to prepare the concentration, one-cycle took 18 minutes, two-cycle took 35 minutes.

In comparison of total procedure time between other methods, our method showed relatively good results. In addition, our method shows the lowest cost among the methods and no infection-related complications were observed.

\section{REFERENCES}

1. Kim SJ, Choi WI, Lee BI, et al. The effect of Platelet-rich plasma (PRP) on the survival of the autologous fat graft. J Korean Soc Plast Reconstr Surg 2007;34:291-7.

2. Eppley BL, Pietrzak WS, Blanton M. Platelet-rich plasma: a review of biology and applications in plastic surgery. Plast Reconstr Surg 2006; 118:147e-59e.

3. Anitua E, Andia I, Ardanza B, et al. Autologous platelets as a source of proteins for healing and tissue regeneration. Thromb Haemost 2004; 91:4-15.

4. Li H, Li B. PRP as a new approach to prevent infection: preparation and in vitro antimicrobial properties of PRP. J Vis Exp 2013;74:e50351.

5. Eppley BL, Woodell JE, Higgins J. Platelet quantification and growth factor analysis from platelet-rich plasma: implications for wound healing. Plast Reconstr Surg 2004;114:1502-8.

6. Marques LF, Stessuk T, Camargo IC, et al. Platelet-rich plasma (PRP): methodological aspects and clinical applications. Platelets 2015;26:10113.

7. Bielecki TM, Gazdzik TS, Arendt J, et al. Antibacterial effect of autologous platelet gel enriched with growth factors and other active substances: an in vitro study. J Bone Joint Surg Br 2007;89:417-20.

8. Moojen DJ, Everts PA, Schure RM, et al. Antimicrobial activity of platelet-leukocyte gel against Staphylococcus aureus. J Orthop Res 2008;26: 404-10.

9. Bielecki T, Gazdzik TS, Szczepanski T. Benefit of percutaneous injection of autologous platelet-leukocyte-rich gel in patients with delayed union and nonunion. Eur Surg Res 2008;40:289-96.

10. Everts PA, Devilee RJ, Brown Mahoney C, et al. Exogenous application of platelet-leukocyte gel during open subacromial decompression contributes to improved patient outcome. A prospective randomized double-blind study. Eur Surg Res 2008;40:203-10.

11. Cieslik-Bielecka A, Bielecki T, Gazdzik TS, et al. Autologous platelets and leukocytes can improve healing of infected high-energy soft tissue injury. Transfus Apher Sci 2009;41:9-12.

12. Li GY, Yin JM, Ding H, et al. Efficacy of leukocyte- and platelet-rich plasma gel (L-PRP gel) in treating osteomyelitis in a rabbit model. J Orthop Res 2013;31:949-56.

13. Akhundov K, Pietramaggiori G, Waselle L, et al. Development of a cost-effective method for platelet-rich plasma (PRP) preparation for topical wound healing. Ann Burns Fire Disasters 2012;25:207-13.

14. Pallua N, Wolter T, Markowicz M. Platelet-rich plasma in burns. Burns 2010;36:4-8.

15. Arteriocyte Medical Systems Inc. MAGELLAN ${ }^{\circledR}$ autologous platelet separator [Internet]. Hopkinton, MA: Arteriocyte Medical Systems Inc.; 2013 [cited 2014 January 5]. Available from: http://www.arteriocyte.com/magellanreg-autologous-platelet-separator.html. 